\title{
Artigo Original \\ A INFLUÊNCIA DA ALTURA DO STEP NO EXERCÍCIO DE SUBIDA POSTERIOR: ESTUDO ELETROMIOGRÁFICO EM INDIVÍDUOS SADIOS E PORTADORES DA SÍNDROME DA DOR FEMOROPATELAR
}

\section{STEP HEIGHT INFLUENCE ON BACKWARD STEP-UP EXERCISE: AN ELECTROMYOGRAPHIC STUDY IN HEALTHY INDIVIDUALS AND IN THOSE WITH PATELLOFEMORAL PAIN SYNDROME}

\author{
Flávio Pulzatto ${ }^{1}$, Karina Gramani-Say', Ana Cristina Barroso de Siqueira², Gillmar Moraes Santos ${ }^{3}$, \\ Débora Bevilaqua-Grossi ${ }^{4}$, Anamaria Siriani de Oliveira ${ }^{4}$, Vanessa Monteiro-Pedro ${ }^{5}$.
}

\begin{abstract}
RESUMO
O objetivo deste estudo foi avaliar a influência da altura de um step na atividade elétrica dos músculos vasto medial oblíquo (VMO), vasto lateral longo (VLL) e vasto lateral oblíquo (VLO) no exercício de subida posterior. Participaram do estudo 27 indivíduos do sexo feminino, sendo 15 sadios (21,13 $\pm 2,17$ anos) e 12 portadores da Síndrome da Dor Femoropatelar-SDFP (21,08 $\pm 2,31$ anos). A atividade elétrica foi captada por eletrodos de superfície. Os indivíduos subiram o step em duas alturas diferentes $\left(45^{\circ}\right.$ e $75^{\circ}$ de flexão do joelho) no sentido posterior. A integral do sinal elétrico normalizado pela média das repetições foi utilizada para o estudo da relação de ativação VMO:VLO e VMO:VLL. A ANOVA two-way e o post hoc de Duncan $(p \leq 0,05)$ mostraram que no grupo SDFP, os valores da relação VMO:VLO e VMO:VLL foram maiores no ângulo de $45^{\circ}$ do que a $75^{\circ}$. No grupo Controle, a relação VMO:VLO foi maior a $45^{\circ}$ que a $75^{\circ}$ enquanto que a relação VMO:VLL foi maior no ângulo de $75^{\circ}$. Os resultados deste estudo sugerem que o exercício de subida posterior no step a $45^{\circ}$ está indicado no tratamento da SDFP, pois ativou mais seletivamente o músculo VMO
\end{abstract}

Descritores: Joelho; Dor; Eletromiografia; Músculo; Exercício.

\section{INTRODUÇÃO}

A Síndrome da Dor Femoropatelar (SDFP) é definida como uma desordem dolorosa da articulação do joelho resultante do mau alinhamento patelar ${ }^{(1)}$. Seu principal sintoma é a dor difusa na região anterior do joelho ou retropatelar cujos efeitos causam prejuízos funcionais aos indivíduos acometidos ${ }^{(1)}$. De início insidioso, atinge principalmente mulheres jovens, adolescentes e atletas de ambos os sexos ${ }^{(2)}$ sendo que o quadro doloroso pode ser intensificado após a realização de atividades como: correr, agachar-se, subir e descer escadas ou ladeiras, permanecer muito tempo sentado ou levantar-se da posição sentada(3).

A etiologia da SDFP não está bem estabelecida, porém, desequilíbrios entre os músculos estabilizadores da patela têm sido sugeridos como um dos principais fatores desencadeadores da SDFP(3). Entretanto, na literatura ainda há controvérsias sobre a ação muscular na estabilização patelar, enquanto alguns trabalhos sugerem haver desequilíbrios na amplitude da atividade elétrica dos músculos vasto medial oblíquo $(\mathrm{VMO})$ e vasto lateral $(\mathrm{VL})^{(4,5)}$, outros não observaram este fato ${ }^{(2,6)}$. O músculo vasto lateral oblíquo (VLO), a partir de estudos anatômicos ${ }^{(7)}$ e eletromiográficos ${ }^{(8)}$,

\begin{abstract}
SUMMARY
The aim of this study was to evaluate the influence of the step height in the electric activity of the vastus medialis oblique (VMO), vastus lateralis longus (VLL) e vastus lateralis oblique (VLO) on backward step-up exercise. Twenty-seven females, being 15 healthy (21.13 \pm 2.17 years old) and 12 with Patellofemoral Pain Syndrome PPS (21.08 \pm 2.31 years old) have participated in the study. The electric activity was recorded by surface electrodes. The volunteers performed the backward step-up exercise in two different heights: with the knee joint flexion at $45^{\circ}$ and at $75^{\circ}$. The whole electric signal normalized by the average of repetitions was used to study the VMO:VLO and VMO:VLL activation ratio. The two-way ANOVA and Duncan post hoc $(p \leq 0.05)$ showed that in the PPS group, the VMO: VLO and VMO: VLL ratio was greater at $45^{\circ}$ than at $75^{\circ}$ angle. In the Control group, the VMO: VLO ratio was greater at $45^{\circ}$ than at $75^{\circ}$, while the VMO: VLL ratio was greater at $75^{\circ}$. The results of this study suggest that backward step-up exercise at $45^{\circ}$ can be used for PPS treatment programs because it activated the VMO muscle in a more selective way.
\end{abstract}

Keywords: Knee; Pain; Electromyography; Muscle; Exercise.

passou a ser considerado importante para a estabilização patelar como antagonista ao VMO tracionando lateralmente a patela ${ }^{(9)}$. No tratamento conservador da SDFP, os programas objetivam o fortalecimento do quadríceps e mais seletivamente do músculo VMO, considerado essencial na manutenção do alinhamento patelar ${ }^{(6)}$. Dos exercícios recomendados, o step está indicado nas fases intermediária e final da reabilitação da SDFP, pois simulam atividades funcionais e permitem a co-contração muscular, resultando em maior estabilidade articular ${ }^{(3,10)}$.

Os exercícios no step podem ser realizados em diferentes alturas permitindo a obtenção de diferentes angulações de flexão do joelho. Do mesmo modo, os exercícios no step podem ser realizados de maneira concêntrica (subida) ou excêntrica (descida), e ainda, no sentido anterior, lateral ou posterior. O step posterior é indicado no tratamento da SDFP(11), porém há uma escassez de trabalhos que tenham analisado o efeito deste exercício na atividade elétrica muscular.

Na literatura consultada, somente o trabalho de Cabral e MonteiroPedro ${ }^{(12)}$ controlou a altura do step com o joelho flexionado a $75^{\circ}$, os autores avaliaram a atividade elétrica dos músculos VMO, VLL

Trabalho realizado no Laboratório de Avaliação e Intervenção em Ortopedia e Traumatologia (LAIOT) do Departamento de Fisioterapia da Universidade Federal de São Carlos - UFSCar.

Endereço para correspondência: Rua Santos Dumont, 1490 - CEP 16200-330 Centro - Birigui - SP - e-mail: flaviopul@yahoo.com.br.

1. Mestre em Fisioterapia - UFSCar

2. Graduanda do curso de Fisioterapia - UFSCar
3. Doutorando do Programa de Pós-Graduação em Fisioterapia - UFSCar

4. Poutorando do Programa de Pós-Graduaçáo em Fisioterapia - UFSCar

5. Professora, Doutora do Departamento de Biomecânica, Medicina e Reab

Trabalho recebido em: 24/01/05 aprovado em 01/07/05 
e VLO nos exercícios de subida e descida, tanto anterior quanto posterior em 18 indivíduos portadores de SDFP, os resultados mostraram que o músculo VMO foi mais ativo que o VLO e o VLL em todos os exercícios. Entretanto, não foram encontrados na literatura trabalhos comparando diferentes alturas de step e suas implicações na atividade elétrica.

Desse modo, o objetivo deste estudo foi investigar o efeito de diferentes alturas de step ( $45^{\circ}$ e $75^{\circ}$ de flexão do joelho) na atividade elétrica dos músculos VMO, VLO e VLL em indivíduos normais e portadores de SDFP. Para isso foram analisadas as relações de atividade VMO:VLO e VMO:VLL nos exercícios de subida posterior em um step de altura regulável.

\section{MATERIAL E MÉTODOS}

\section{Indivíduos}

Participaram do estudo 27 voluntários do sexo feminino na faixa etária de 18 a 30 anos, não atletas, divididos em dois grupos. O grupo Controle foi composto por 15 indivíduos $(21,13 \pm 2,17$ anos) sem história de cirurgia, dor, trauma ou lesão no sistema osteomioarticular nos membros inferiores.

O grupo portador de SDFP foi composto por 12 indivíduos $(21,08$ $\pm 2,31$ anos) que apresentaram: (1) Dor em pelo menos 3 das seguintes atividades: agachar por tempo prolongado, subir ou descer escadas, ajoelhar, correr, permanecer muito tempo sentado, contrair isometricamente o músculo quadríceps e praticar esporte. (2) Intensidade de dor no mínimo de $2 \mathrm{~cm}(0-10 \mathrm{~cm})$ na Escala Visual Analógica (EVA) no período da última semana durante a realização das atividades citadas acima e ainda, referir dor de qualquer grandeza durante a realização dos seguintes testes funcionais: descida de um step de $25 \mathrm{~cm}$ de altura e agachamento bipodal com os joelhos fletidos a $90^{\circ}$, ambos realizados durante 30 segundos.

Todos os voluntários assinaram um Termo de Consentimento Formal e Esclarecido para a participação na pesquisa que foi aprovada pelo Comitê de Ética em Pesquisa da Universidade Federal de São Carlos.

\section{Instrumentos}

A atividade elétrica dos músculos VMO, VLL e VLO foi captada por meio de eletrodos ativos diferenciais simples de superfície $(\mathrm{Ag} / \mathrm{AgCl})$ conectados a um eletromiógrafo EMG-8 (EMG System do Brasil). A taxa de amostragem foi de $2000 \mathrm{~Hz}$ e utilizou-se um filtro passa-banda de 20 a $500 \mathrm{~Hz}$. Para aquisição dos dados foi utilizado o software AqDados 7.02.06.

O step utilizado neste trabalho consistiu de um degrau de madeira de $49 \mathrm{~cm}$ de altura, $68 \mathrm{~cm}$ de largura e $40 \mathrm{~cm}$ de profundidade e possui um mecanismo tipo gaveta que permite a regulagem de sua altura com precisão de $1,5 \mathrm{~cm}{ }^{(12)}$. Para a obtenção dos ângulos de $45^{\circ}$ e $75^{\circ}$ de flexão do joelho, o step foi regulado em média a $11 \mathrm{~cm}$ e $21 \mathrm{~cm}$, respectivamente. Um metrônomo (Witner) regulado em 63 batidas por minuto auxiliou os voluntários no controle do tempo de execução dos exercícios de subida posterior no step. Utilizou-se um eletrogoniômetro, conectado ao eletromiógrafo e fixado na articulação do joelho estudado para monitorar a amplitude de flexão no momento do exercício.

\section{Procedimentos}

Após tricotomia e limpeza da pele com álcool $70 \%$, os eletrodos foram fixados sobre a pele do voluntário com adesivo dupla-face especial para eletromiografia no ventre de cada músculo e com as superfícies de detecção no sentido perpendicular às fibras musculares ${ }^{(10)}$.

Para a fixação dos eletrodos, inicialmente foi traçada uma linha que vai da espinha ilíaca ântero-superior ao centro da patela, esta linha serviu de referência para a medida dos ângulos de inclinação de todos os eletrodos. Para o músculo VMO, o eletrodo foi fixado a $4 \mathrm{~cm}$ acima da borda súpero-medial da patela e inclinação de $55^{\circ(3)}$. No músculo VLL o eletrodo foi fixado $15 \mathrm{~cm}$ acima da borda súpero-lateral da patela com inclinação de $13,6^{\circ(9)}$ e no músculo VLO, o eletrodo foi fixado no meio do ventre muscular a partir da localização do epicôndilo femoral lateral com inclinação lateral de $50,4^{\circ(9)}$. Foi utilizado também um eletrodo de referência tipo garra com superfície de metal de $2,5 \times 3,5 \mathrm{~cm}$ untado com gel condutor, fixado na região distal da perna do voluntário, próximo a articulação do tornozelo homolateral ao estudado.

Os voluntários, auxiliados pelo metrônomo, realizaram 3 repetições de subida posterior no step em duas angulações diferentes. Para isso, a altura do step foi regulada para cada voluntário de modo que, no momento da subida no step, o ângulo de flexão da articulação do joelho ficasse em $45^{\circ}$ na primeira série e em $75^{\circ}$ na segunda série de exercícios.

\section{Exercícios e Análise do Sinal}

Após alongamento e aquecimento dos principais grupos musculares dos membros inferiores, foram realizadas três repetições de subida posterior no step a $45^{\circ}$ e $75^{\circ}$, num total de 6 repetições.

O voluntário foi posicionado de costas para o step e após o comando verbal: "atenção, prepare, pode subir", realizava a subida posterior no step, iniciando o exercício com o membro a ser estudado seguido do contralateral como mostrado na Figura 1.

Os sinais eletromiográficos da contração concêntrica (subida) foram processados por uma rotina do software Matlab 6.1 que calculou o valor da integral da área abaixo da envoltória do sinal elétrico após filtragem e retificação por onda completa. Os dados foram normalizados pelo valor médio das envoltórias das três repetições de cada voluntário.

A partir dos valores da integral do sinal de cada músculo, foram calculados os valores da relação entre os músculos VMO:VLO e VMO:VLL

\section{Análise Estatística}

Para a comparação entre os grupos Controle e SDFP bem como entre as diferentes alturas do step, foi utilizado a análise de variância (ANOVA two way) e post hoc de Duncan, ambos considerando $p \leq 0,05$.

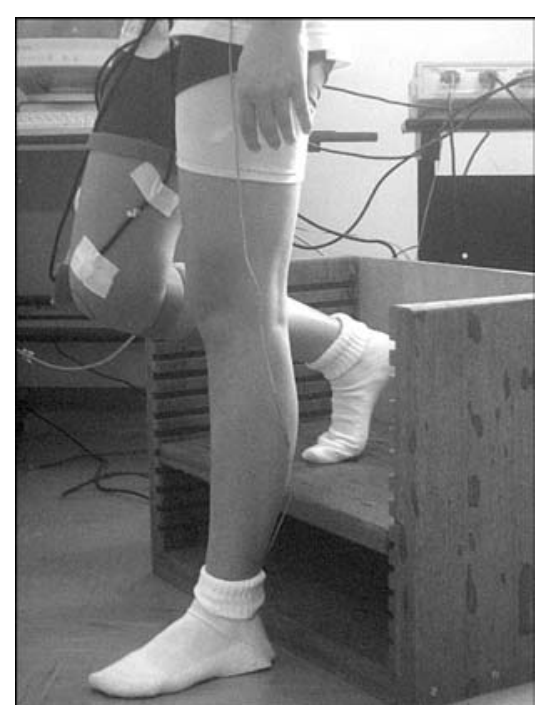

Figura 1 - Voluntário realizando o exercício de subida posterior no step de altura regulável.

\section{RESULTADOS}

A análise das relações de atividade elétrica VMO:VLO e VMO:VLL mostrou haver diferenças significativas entre os ângulos (alturas do step) de $45^{\circ}$ e $75^{\circ}$ tanto no grupo Controle $(p=0,00)$ quanto no grupo SDFP $(p=0,00)$ (Tabela 1).

O grupo SDFP apresentou valores da relação muscular maiores no step a $45^{\circ}$ quando comparados ao step de $75^{\circ}$, tanto para a relação VMO:VLO quanto para a VMO:VLL (Tabela 1).

No grupo Controle a relação VMO:VLO foi maior no ângulo de $45^{\circ}$ enquanto que a relação VMONLL foi maior no step a $75^{\circ}$ (Tabela 1).

$\mathrm{Na}$ análise intermusculo, a relação VMO/ VLL foi maior que a relação VMO/VLO tanto no grupo Controle $(p=0,00)$ quanto no grupo SDFP $(p=0,00)$ em ambas as angulações estudadas (Tabela 1). A análise intergrupos mostrou não haver diferenças nos valores da relação muscu- 
lar entre os grupos Controle e $\operatorname{SDFP}(p=0,53)$ em ambas as alturas de step.

\section{DISCUSSÃO}

Nossos resultados mostraram que o exercício realizado no step a $45^{\circ}$ recrutou de maneira mais acentuada o músculo VMO em relação aos músculos VLO e VLL quando comparado ao ângulo de $75^{\circ}$ em ambos os grupos.

Na prática clínica do tratamento da SDFP, objetiva-se o fortalecimento seletivo do VMO com o propósito de manter o equilíbrio das forças mediais e laterais que atuam sobre a patela uma vez que este músculo é seletivamente atrofiado nas situações resultantes em SDFP(13). Além disso, os exercícios em cadeia cinética fechada (CCF) como o step causam menor estresse femoropatelar sendo os mais indicados no tratamento da SDFP(9).

Desse modo, os exercícios de subida posterior no step realizados com a articulação do joelho a $45^{\circ}$ estão indicados no tratamento da SDFP pois, proporcionaram maior vantagem do músculo VMO em relação aos seus antagonistas. Além disso, a $45^{\circ}$ de flexão, a articulação femoropatelar apresenta-se estável e há grande congruência da patela no sulco troclear ${ }^{(14)}$ o que corrobora a indicação desta angulação para a realização dos exercícios no step.

Cabral e Monteiro-Pedro ${ }^{(12)}$ também observaram maior atividade do músculo VMO em relação ao VLO e VLL em indivíduos portadores da SDFP durante exercícios de subida e descida no step a $75^{\circ}$ de flexão do joelho. Os autores relatam que os exercícios de step posterior recrutaram de maneira mais intensa o músculo VMO e sugerem a indicação deste exercício na reabilitação da SDFP. Os valores da relação muscular entre 1 e 1,3 encontrados no presente estudo estão de acordo com trabalhos prévios tanto com indivíduos sadios quanto portadores de SDFP em CCF $(5,4)$ em ambas as angulações. Estes valores indicam que o músculo VMO sempre foi mais ativado que seus antagonistas. Por outro lado, Tang et al. ${ }^{(15)}$ encontraram valores da relação VMO:VLL menores que 1 durante o exercício de agachamento, tanto a $45^{\circ}$ quanto a $75^{\circ}$ de flexão do joelho em indivíduos sadios e portadores de SDFP. Estes valores indicam maior ativação do músculo VL em comparação ao VMO, não concordando com nossos resultados em ambas as angulações do joelho.

Como relatado anteriormente, não foram encontrados na literatura estudos que tenham investigado o efeito da altura do step na atividade elétrica dos estabilizadores patelares. Acreditamos que esta metodologia permite que os voluntários sejam submetidos a valores proporcionais de compressão e estresse patelares independentemente da estatura de cada indivíduo. Assim, o controle do ângulo de flexão do joelho a partir da regulagem da altura do step, padroniza a comparação entre grupos e exercícios. Em relação ao músculo VLO, com exceção ao trabalho de Cabral e Monteiro-Pedro(12), não foram encontrados trabalhos na literatura investigando sua atividade elétrica em tarefas funcionais (como o step, por exemplo).

Nossos resultados mostraram que no step a $75^{\circ}$, o músculo VLO apresentou valores de atividade próximos aos do músculo VMO em ambos os grupos, sendo que este padrão não se repetiu para o músculo VLL. Este comportamento sugere que o músculo VLO pode ser recrutado de maneira mais acentuada quanto maior o ângulo de flexão da articulação do joelho, participando mais ativamente na lateralização patelar que o músculo VLL.

Um dado importante do nosso trabalho é o fato de o grupo controle apresentar maior valor de relação VMO:VLL a $75^{\circ}$ não pode ser explicado apenas pela eletromiografia, mas outros fatores podem estar envolvidos. Porém, como observado, os outros valores da relação muscular foram significativamente maiores no step a $45^{\circ}$, principalmente no grupo SDFP, de modo a confirmar a indicação desta angulação no tratamento desta população. Nossos resultados, assim como trabalhos prévios ${ }^{(2,6)}$, não evidenciaram diferenças na relação da atividade elétrica entre os grupos estudados. Isto pode indicar que outros fatores, anatômicos ou biomecânicos, possam estar relacionados com a presença da SDFP e não somente o desequilíbrio na ação muscular ${ }^{(3)}$.

\section{CONCLUSÕES}

Nossos resultados mostraram que as relações VMO:VLO e VMO: VLL foram significativamente maiores no exercício de step com $45^{\circ}$ de flexão do joelho. Assim, no tratamento conservador da SDFP, este exercício deve ser preferencialmente utilizado, pois recruta de maneira seletiva o músculo VMO em relação aos seus antagonistas favorecendo a estabilidade patelar nos indivíduos portadores da SDFP. Além disso, o exercício no step a $75^{\circ}$ potencializou a ativação do músculo VLO em ambos os grupos, favorecendo o deslocamento lateral da patela, sendo contra-indicado aos portadores da SDFP.

\section{REFERÊNCIAS BIBLIOGRÁFICAS}

1. Thomeé R, Augustsson J, Karlsson J. Patellofemoral pain syndrome. A review of current issues. Sports Med 1999; 28:245-62.

2. Cerny K. Vastus medialis oblique/vastus lateralis muscle activity ratios for selected exercises in persons with and without patellofemoral pain syndrome. Phys Ther 1995; 75:672-83.

3. Cowan SM, Bennell KL, Hodges PW, Corssley KM, McConnell J. Delayed onset of electromyographic activity of vastus medialis obliquus relative to vastus lateralis in subjects with patellofemoral pain syndrome. Arch Phys Med Rehabil 2001; 82:183-9.

4. Souza DR, Gross MT. Comparison of vastus medialis obliquus: vastus lateralis muscle integrated electromyographic ratios between healthy subjects and patients with patellofemoral pain. Phys Ther 1991; 71:310-20.

5. Taskiran E, Dinedurga Z, Yagiz A, Uludag B, Ertekin C, LökV. Effect of the vastus medialis obliquus on the patellofemoral joint. Knee Surg Sports Traumatol Arthrosc 1998: 6:173-80.

6. Sheehy P, Burdett RG, Irrgang JJ, Vanswearingen J. An electromyographic study of vastus medialis oblique and vastus lateralis activity while ascending and descending steps. J Orthop Sports Phys Ther 1998; 27:423-9.

7. Hallisey MJ, Doherty N, Bennett WF, Fulkerson JP. Anatomy of the junction of the vastus lateralis tendon and the patella. J Bone Joint Surg Am 1987; 69:545-9.

8. Morrish GM, Woledge RC. A comparison of the activation of muscles moving the patella in normal subjects and in patients with chronic patellofemoral problems. Scand J Rehabil Med 1997; 29:43-8.

9. Bevilaqua-Grossi D, Monteiro-Pedro V, Bérzin F. Análise funcional dos estabilizadores patelares. Acta Ortop Bras 2004; 12: 99-104

10. Monteiro-Pedro V, Vitit M, Bérzin F, Bevilaqua-Grosso D. Electromyographic activity of vastus medialis oblique muscle in step-up and step-down exercises. Brazilian J Morphol Sci 1997; 14:19-23

11. McConnel J. The management of chondromalacia patellae: a long-term solution. Aust J Physioth 1986; 32:215-23.

12. Cabral CMN, Monteiro-Pedro V. Efeito dos exercícios em cadeia cinética fechada realizados no step na atividade elétrica dos componentes medial e lateral do músculo quadríceps femoral. Dissertação. São Carlos: Universidade Federal de São Carlos; 2001

13. Grabiner MD, Koh TJ, Draganich LF. Neuromechanics of the patellofemoral joint. Med Sci Sports Exerc 1994; 26:10-21.

14. Manske RC, Davies GJ. A nonsurgical approach to examination and treatment of the patellofemoral joint. Part 1: examination of the patellofemoral joint. Crit Rev Phys Rehabil Med. 2003; 15:141-66

15. Tang SF, Chen CK, Hsu R, Chou SW, Hong WH, Lew HL. Vastus medialis obliquus and vastus lateralis activity in open and closed kinetic chain exercises in patients with patellofemoral pain syndrome: an electromyographic study. Arch Phys Med Rehabil $2001 ; 82: 1441-5$. 